\title{
Mamíferos del departamento del Atlántico, Colombia
}

\section{Mammals of the Department of Atlántico, Colombia}

\author{
Luis José Avendaño-Maldonado ${ }^{\circ} \square$, Miguel Ángel Camargo-Alarcón ${ }^{\circledR} \nabla$, \\ Rafael Borja-Acuña $\square$, Julio J. Chacón-Pacheco $\square$
}

\section{Resumen}

Presentamos el primer listado taxonómico de los mamíferos silvestres del departamento del Atlántico, en la costa Caribe de Colombia. Registramos 92 especies, distribuidas en 11 órdenes, 37 familias y 74 géneros, lo cual representa el 16.5 \% de la mastofauna documentada para Colombia. Encontramos tres especies endémicas de Colombia, Cebus versicolor, Saguinus oedipus y Pattonomys semivillosus, 10 especies en alguna categoría nacional de amenaza, 13 especies en alguna categoría global y cuatro categorizadas como Datos Insuficientes. La riqueza de mamíferos es baja, comparada con la de otros departamentos del país, pero podría estar subestimada, debido al bajo esfuerzo de muestreo y la falta de exploración en diversas áreas del departamento.

Palabras clave. Caribe colombiano. Chiroptera. Diversidad taxonómica. Rodentia. Serranía de Piojó.

\begin{abstract}
We presents the first taxonomic list of wild mammals for the Atlántico department, in the Caribbean coast of Colombia. We recorded 92 species distributed in 11 orders, 37 families, and 74 genera, which represents 16.5 $\%$ of the mammal fauna recorded in Colombia. We found three species endemic to Colombia, Cebus versicolor, Saguinus oedipus, and Pattonomys semivillosus, 10 species in some national category of threat, 13 in any global category, and four Data Defficient. The representativeness of mammals is low, compared to other departments in the country, but it might be underestimated, due to low sampling effort and lack of exploration in various areas of the department.
\end{abstract}

Keywords. Colombian Caribbean. Chiroptera. Taxonomic diversity. Rodentia. Serranía de Piojó. 


\section{Introducción}

Colombia tiene una gran diversidad biológica, principalmente por su historia geológica y por la variedad de ambientes que este posee (Hernández-Camacho et al., 1992). Esto se ve reflejado en la diversidad de mamíferos, de los que hay en Colombia 528 especies, distribuidas en 14 órdenes y 49 familias (Sociedad Colombiana de Mastozoología, 2019). En las últimas décadas se han publicado varias listas nacionales y regionales de los mamíferos de Colombia. (Cuervo-Díaz et al., 1986; Alberico et al., 2000; Solari et al., 2013; Ramírez-Chaves et al., 2016). Estas listas constituyen una herramienta necesaria para la toma de decisiones sobre la organización del territorio y la conservación de la biodiversidad (Solari et al., 2013). No obstante, algunas áreas carecen de estudios de referencia locales, lo que dificulta el intercambio de información biológica sobre mamíferos en la región(Adler et al., 1997; Díaz-Pulido et al., 2014; Racero-Casarrubia et al., 2015). Uno de los departamentos más rezagados en materia del conocimiento de mamíferos ha sido el Atlántico.

El departamento del Atlántico está ubicado al norte de Colombia, dentro de la provincia del Cinturón Árido-Pericaribeño (Hernández-Camacho et al., 1992), y a pesar de ser una de las divisiones político-administrativas con menor extensión $\left(3382 \mathrm{~km}^{2}\right)$, y caracterizarse por un relieve mayormente uniforme, alberga un enclave azonal de montañas bajas que corresponde al distrito de la serranía de Piojó e Hibácharo. La zona de vida nativa del territorio corresponde a bosque seco tropical (bs-T; Holdridge, 1982); sin embargo, dada la constante expansión de la frontera agrícola y ganadera, este ecosistema se ha reducido notablemente, reduciendose a un bs-T de tipo relictual (8\%) (Rodríguez et al., 2012; García et al., 2014). Adicionalmente, hacia el norte del departamento y a lo largo de la línea de costa se encuentran ecosistemas marino-costeros, como lagunas costeras, manglares, playas y dunas, que se ven amenzadas por procesos de sedimentación-colmatación, erosión y contaminación por afluentes procedentes del continente (INVEMAR, 2007).

Los grupos aborígenes Mokaná, que habitaron el territorio atlanticense, representaron hace mil años, en forma de arte rupestre, especies nativas como el mono aullador (Alouatta seniculus) y el oso hormiguero (Tamandua mexicana), en el petroglifo de Piedra Pintada del municipio de Tubará en la subregión Costera (Yance-Pérez, 1990; Baquero-Montoya \& De La Hoz-Siegler, 2011). Posteriormente, en el desarrollo de los primeros estudios científicos para el departamento, los investigadores
Leo E. Miller y Howarth S Boyle, recolectaron en 1915 dos especímenes de Carollia perspicillata y uno de Sylvilagus floridanus, cerca de Barranquilla. Esta fue una de las expediciones realizadas por el American Museum of Natural History de Nueva York, dirigidas por el ornitólogo Frank Chapman (Allen, 1916; Quintero-Toro, 2012). Posteriormente, Phillip Hershkovitz (1948; 1949) recolectó en el departamento alrededor de 32 especímenes, entre zarigüeyas (Didelphis marsupialis), murciélagos (Glossophaga soricina y Molossus molossus) y un roedor (Pattonomys semivillosus), que reposan en el Field Museum of Natural History de Chicago. Hershkovitz probablemente ha sido uno de los investigadores que más aportó al conocimiento de mamíferos en el Atlántico y de Colombia en el siglo XX (Alberico et al., 2000).

Para Atlántico no se cuenta con una lista actualizada de los mamíferos silvestres, más allá de lo documentado por Solari et al. (2013), quienes registran la presencia de 80 especies en el territorio del departamento. La falta de conocimiento sobre la mastofauna del Atlántico a causa de los pocos estudios, los inventarios no publicados y la falta de exploración de algunas áreas, agregado a la ocurrencia de ecosistemas amenazados, hacen prioritario establecer el estado de conservación de este grupo (Muñoz-Saba \& Hoyos, 2012; Díaz-Pulido et al., 2014).

\section{Materiales y métodos}

Área de estudio. El departamento del Atlántico se sitúa al norte del territorio colombiano, en el margen izquierdo del río Magdalena entre los $10^{\circ} 15^{\prime} 36^{\prime \prime} \mathrm{N}-11^{\circ}$ $06^{\prime} 37^{\prime \prime} \mathrm{N}$, y $74^{\circ} 42^{\prime} 47^{\prime \prime} \mathrm{O}-75^{\circ} 16^{\prime} 34^{\prime \prime} \mathrm{O}$, y tiene $74 \mathrm{~km}$ de litoral en el mar Caribe. En el departamento se han establecido áreas de interés ambiental bajo el criterio de Sistemas Ambientales Estratégicos, en los que se definen tres zonas: río Magdalena, zona Costera y zona Canal del Dique (Figura 1; Gobernación del Atlántico, 2012). A lo largo del margen del río Magdalena predominan las tierras bajas inferiores a 15 m s.n.m, en las cuales abundan llanuras aluviales, y ciénagas que se comunican entre sí mediante numerosos caños. En la zona Canal del Dique se encuentra el espejo de agua más grande del departamento conocido como el embalse del Guájaro. Hacia el occidente, en la zona Costera, se extiende una zona montañosa que corresponde a la prolongación de la Serranía de San Jerónimo, la cual se divide en dos tramos principales de poca elevación, la Serranía del Caballo y la Serranía de Piojó, cuyos puntos más altos están a 520 y 510 m s.n.m, respectivamente (Dugand, 1947; Gobernación del Atlántico, 2012). 


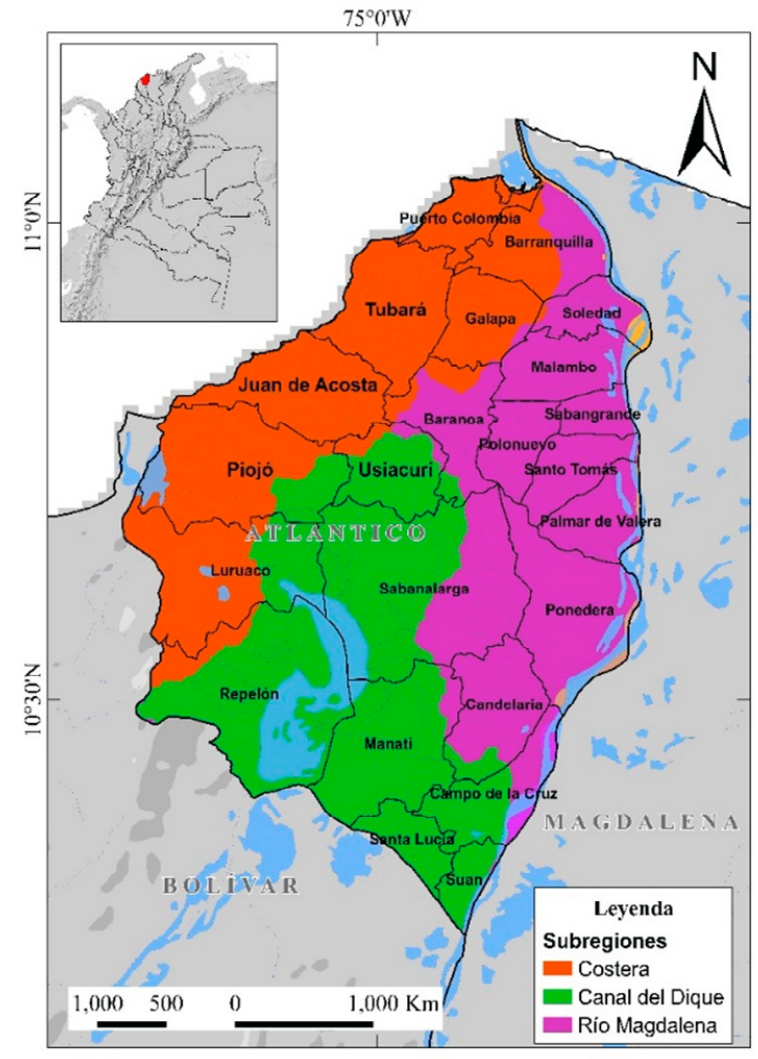

Figura 1. Ubicación geográfica del departamento de Atlántico, Colombia.

Figure 1. Geographic location of the Department of Atlántico, Colombia.

El clima en el departamento del Atlántico se caracteriza por dos temporadas bien marcadas, que responden a un régimen pluviométrico (Castilla-Consuegra, 2013), una temporada de lluvias y otra seca. La temporada de lluvias va desde abril hasta noviembre, interrumpida brevemente en junio y julio por un "veranillo"; la temporada seca inicia a principios de diciembre y se extiende hasta febrero y marzo (Guzmán et al., 2014).

Métodos. La presente lista se realizó a partir de la revisión del material biológico presente en el Museo de Colecciones Biológicas de la Universidad del Atlántico (UARC). Además, se consultó y recopiló información a partir de las plataformas virtuales del GBIF (Global Biodiversity Information Facility), y el SiB (Sistema de Información sobre Biodiversidad de Colombia), de las cuales se obtuvieron datos de especímenes depositados en el Instituto Alexander von Humboldt (IAvH), Instituto de Ciencias Naturales de la Universidad Nacional de Colombia (ICN),
Field Museum of Natural History (FMNH), University of Kansas Biodiversity Institute, Mammalogy Collection (KUM), Museum of Comparative Zoology (MCZ), Muséum d'Histoire Naturelle de la Ville de Genève (MHNG), Museum of Southwestern Biology (MSB), Museum of Vertebrate Zoology, University of California, Berkeley (MVZ), Sam Noble Oklahoma Museum of Natural History (OMNH), Royal Ontario Museum (ROM), Yale Peabody Museum of Natural History (YPM) y Zoologische Staatssammlung München (ZSM).

Adicionalmente, se realizó una búsqueda de literatura científica mediante los buscadores Google Académico, Science Direct, Scopus y SciELO, utilizando como palabras clave, "Atlántico", "Colombia", "mamíferos" y combinaciones con cada uno de los órdenes de mamíferos presentes en el país. También se incluyeron registros de observaciones, fotografías y recolectas realizadas por los autores en la ejecución de salidas de campo.

A partir de la información obtenida se elaboró una lista de órdenes, familias y especies de mamíferos, que sustentan la distribución de cada taxón en el departamento del Atlántico.

Para este trabajo se sigue el tratamiento taxonómico de Burgin et al. (2018) y la base de datos Mammal Diversity Database. Para fines prácticos los órdenes Artiodactyla y Cetacea se manejan por separado. Dentro de Chiroptera se adopta la propuesta de Gardner (2008); se adopta la propuesta de Baker et al. (2016) para la familia Phyllostomidae, Loureiro et al. (2020) para las especies del género Molossus, y la propuesta de Siles \& Baker (2020) para Micronycteris. Para Rodentia se adopta la propuesta de Patton et al. (2015) y para el orden Pilosa se acepta la familia Choloepodidae (Delsuc et al., 2019).

Finalmente, se consultó el estado de amenaza a nivel nacional (Resolución 1912; Ministerio del Ambiente y Desarrollo Sostenible 2017) y global de acuerdo con The Red List of Threatened Species de la International Union for Conservation of Nature (Version 2020-2).

\section{Resultados}

Se obtuvieron 680 registros, de los cuales 357 (52.5\%) fueron a partir de bases de datos, $193(28.4 \%)$ soportados por especímenes, 119 (17.5\%) de literatura y 11 (1.6\%) registros a partir de observaciones directas en campo. La mayoría de registros provienen de los municipios de Barranquilla (23\%), Piojó (20\%) y Usiacurí (16\%). 
En la presente lista se registraron 92 especies de mamíferos para el departamento del Atlántico, distribuidas en 11 órdenes, 37 familias y 74 géneros (Anexo 1); los municipios de Usiacurí, Piojó y Barranquilla presentan el mayor número de especies, 42,42 y 37, respectivamente. Los municipios de Campo de la Cruz, Candelaria, Malambo, Santo Tomás y Soledad no cuentan con registro de especies (Figura 2). La Zona Costera fue la subregión con mayor riqueza (76 spp.), seguida de la zona Canal del Dique (56 spp.) y la zona Río Magdalena (21 spp.).

Los quirópteros se sitúan como el orden más diverso con 36 especies (39.1\%), seguido de Cetacea y Carnivora con 14 especies cada uno (15.2 \%; Tabla 1). Las familias con mayor riqueza corresponden a Phyllostomidae (Chiroptera) y Delphinidae (Cetacea) con 18 y 8 especies respectivamente.

En el Atlántico se encuentran tres especies endémicas de Colombia: dos primates (Cebus versicolor, Saguinus oedipus) y un roedor (Pattonomys semivillosus). Se registran al menos 10 especies con alguna categoría de amenaza a nivel nacional y 13 especies con alguna categoría de amenaza a nivel mundia; además, hay cuatro especies categorizadas como Datos Insuficientes (Tabla 2).

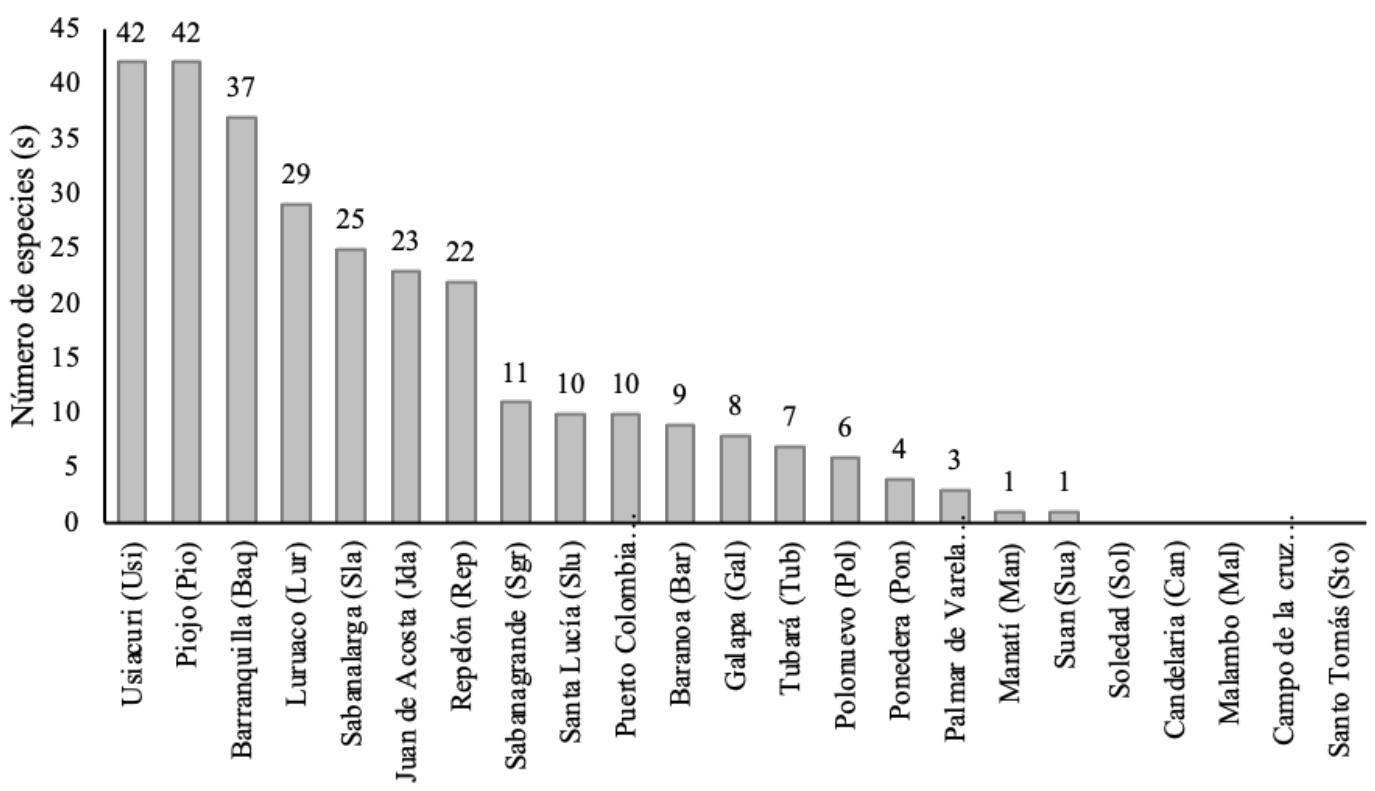

Figura 2. Riqueza de especies de mamíferos por municipios presentes en el departamento de Atlántico, Colombia.

Figure 2. Richness of mammal species by municipalities in the department of Atlántico, Colombia.

Tabla 1. Número de familias, géneros y especies de mamíferos registradas para el departamento del Atlántico y (en paréntesis) para Colombia (Sociedad Colombiana de Mastozoología, 2019).

Table 1. Number of families, genera and species of mammals recorded for the Atlántico department and (in parentheses) for Colombia (Sociedad Colombiana de Mastozoología, 2019).

\begin{tabular}{cccc}
\hline ORDEN & Familias & Géneros & Especies \\
\hline Didelphimorphia & $1(1)$ & $2(13)$ & $2(40)$ \\
\hline Sirenia & $1(1)$ & $1(1)$ & $1(2)$ \\
\hline Cingulata & $2(2)$ & $2(3)$ & $2(6)$ \\
\hline Pilosa & $4(4)$ & $5(5)$ & $5(7)$ \\
\hline
\end{tabular}




\begin{tabular}{cccc}
\hline ORDEN & Familias & Géneros & Especies \\
\hline Primates & $4(5)$ & $5(15)$ & $6(40)$ \\
\hline Lagomorpha & $1(1)$ & $1(1)$ & $1(4)$ \\
\hline Chiroptera & $7(9)$ & $24(70)$ & $36(209)$ \\
\hline Carnivora & $4(6)$ & $12(24)$ & $2(34)$ \\
\hline Artiodactyla & $2(2)$ & $2(5)$ & $14(30)$ \\
\hline Cetacea & $3(5)$ & $11(19)$ & $9(132)$ \\
\hline Rodentia & $8(10)$ & $9(56)$ & $0(3)$ \\
\hline Perissodactyla & $0(1)$ & $0(1)$ & $0(2)$ \\
\hline Paucituberculata & $0(1)$ & $0(1)$ & $0(7)$ \\
\hline Eulipotyphla & $0(1)$ & $0(1)$ & 92 \\
\hline TOTAL & 37 & 74 & \\
\hline
\end{tabular}

Tabla 2. Lista de especies de mamíferos del departamento del Atlántico, Colombia, en alguna categoría de amenaza, según la categorización nacional (Ministerio del Ambiente y Desarrollo Sostenible, 2017) (MADS) y global (IUCN, 2020).

Table 2. List of mammal species in the department of Atlántico, Colombia, in some category of threat according to national (Ministerio del Ambiente y Desarrollo Sostenible, 2017) (MADS) and global (IUCN, 2020) categorization.

\begin{tabular}{|c|c|c|c|c|}
\hline ORDEN & FAMILIA & ESPECIE & MADS & IUCN \\
\hline Artiodactyla & Cervidae & Mazama americana & & Datos Insuficientes \\
\hline \multirow{3}{*}{ Carnivora } & Mustelidae & Lontra longicaudis & Vulnerable & Casi Amenazado \\
\hline & Felidae & Leopardus wiedii & & Casi Amenazado \\
\hline & Felidae & Panthera onca & Vulnerable & Casi Amenazado \\
\hline \multirow{5}{*}{ Cetacea } & Balaenopteridae & Balaenoptera musculus & En peligro & En Peligro \\
\hline & Balaenopteridae & Balaenoptera physalus & En peligro & Vulnerable \\
\hline & Delphinidae & Sotalia guianensis & Vulnerable & Casi Amenazado \\
\hline & Physeteridae & Kogia breviceps & & Datos Insuficientes \\
\hline & Physeteridae & Physeter macrocephalus & Vulnerable & Vulnerable \\
\hline Cingulata & Chlamyphoridae & Cabassous centralis & & Datos Insuficientes \\
\hline Pilosa & Myrmecophagidae & $\begin{array}{l}\text { Myrmecophaga } \\
\text { tridactyla }\end{array}$ & & Vulnerable \\
\hline \multirow{4}{*}{ Primates } & Aotidae & Aotus griseimembra & Vulnerable & Vulnerable \\
\hline & Atelidae & Ateles fusciceps & En peligro & En Peligro Crítico \\
\hline & Callitrichidae & Saguinus oedipus & En Peligro Crítico & En Peligro Crítico \\
\hline & Cebidae & Cebus versicolor & & En Peligro \\
\hline Rodentia & Caviidae & Hydrochoerus isthmius & & Datos Insuficientes \\
\hline Sirenia & Trichechidae & Trichechus manatus & En peligro & Vulnerable \\
\hline
\end{tabular}




\section{Discusión}

La riqueza de mamíferos reportada en este trabajo representa el $16.5 \%$ del total documentado para Colombia (Tabla 1; Sociedad Colombiana de Mastozoología, 2019), la cual es baja comparada con otros departamentos del Caribe colombiano (Muñoz-Saba \& Hoyos, 2012; Racero-Casarrubia et al., 2015) y otras regiones (p. ej., Castaño et al., 2003; 2017; Ramírez-Chaves \& Noguera-Urbano 2010; Ramírez-Chaves \& Pérez 2010; Rojas-Díaz et al., 2012; Ramírez-Chaves et al., 2013). No obstante, se debe considerar que el departamento del Atlántico es el tercero más pequeño en extensión del país $\left(3388 \mathrm{~km}^{2}\right)$, representando apenas el $0.26 \%$ del territorio colombiano y el $2.6 \%$ del Caribe colombiano (Gobernación del Atlántico, 2012), con una baja heterogeneidad del paisaje, en comparación con áreas como la Serranía de San Lucas, en el departamento de Bolívar, el Parque Nacional Natural Paramillo, en Córdoba, y la Sierra Nevada de Santa Marta, en los departamentos de Cesar, La Guajira y Magdalena.

En el departamento del Atlántico se encuentran siete de las nueve familias de murciélagos que habitan en Sudamérica (Díaz et al. 2016), con excepción de Thyropteridae y Furipteridae. Dentro de estas, Phyllostomidae es la más representativa en número de especies (18); esta diversidad puede atribuirse a la variedad de hábitats disponibles potencialmente explotables para los murciélagos, agregado al éxito biológico propio que exhibe este taxón, el cual constituye uno de los grupos más diversos en el Neotrópico (Cirranello et al., 2016). Por otro lado, la baja representatividad de las familias Vespertilionidae y Molossidae puede asociarse con los métodos de estudio que se han usado tradicionalmente para el estudio de los murciélagos en la región, como redes de niebla, que dificultan el registro de especies insectívoras (Pech-Canche et al., 2011).

Cetacea y Carnívora se ubican como segundo grupo con mayor riqueza de especies, 14 cada uno; sin embargo, se sabe muy poco acerca de la dinámica de estos. El número de especies de cetáceos se atribuye a que el departamento se encuentra bañado por el Mar Caribe con 74 $\mathrm{km}$ de costa, donde las especies pueden aprovechar la disponibilidad de alimento, producto de la desembocadura del río Magdalena que ofrece recursos para los peces y otros organismos, las grandes profundidades y la morfología de las bahías que pueden proporcionar áreas aptas para el desarrollo de las diferentes actividades de estas especies (Rincón y Romero, 2012; Alvarado, 2016). Atlántico comprende algunas zonas como las playas de
Puerto Colombia y Tubará, donde los avistamientos de estos cetaceos son muy comunes, principalmente de las especies delfín de naríz de botella (Tursiops truncatus) y el delfín de Guyana (Sotalia guianenesis).

Las 14 especies de carnívoros, representan el 41\% de las especies reportadas para Colombia (Tabla 1); tres de estas están categorizadas como Casi Amenazadas (Tabla 2; Sociedad Colombiana de Mastozoología, 2019). La fragmentación y degradación del hábitat, además del conflicto humano-carnívoro, son unas de las principales fuentes de amenaza hacia estos organismos, que juegan un papel fundamental en el mantenimiento de la biodiversidad y el funcionamiento de los ecosistemas (Morrison et al., 2007; Ritchie et al., 2012; Ripple et al., 2014). Los carnívoros normalmente requieren de grandes áreas en ambientes conservados, lo cual está ligado a factores como el tamaño corporal, demanda metabólica, y tácticas de caza (Carbone et al., 2005). Dada la alta presión ganadera y agrícola en el departamento, las especies dependientes de la cobertura de bosque se encuentran altamente amenazadas. No obstante, el efecto de la presión antrópica varía de acuerdo a la especie, y algunas de estas pueden usar sistemas agroforestales como corredores biológicos (Ferreira et al., 2018), regularmente son aquellas especies generalistas, categorizadas en Preocupación Menor. Es importante implementar y estudiar el uso de sistemas agroforestales en el departamento del Atlántico, además de evaluar el papel que cumplen estos sistemas en la conservación de carnívoros.

A pesar de ser un grupo taxonómicamente estable y de aparente facilidad en la determinación a nivel de especie, el género Procyon (Procyonidae), presenta dificultades en la delimitación de P. cancrivorus y P. lotor (González-Maya et al., 2011). Procyon cancrivorus representa una especie común en el departamento, registrada para nueve municipios y las tres subregiones (Anexo 1); se encuentra asociada tanto a ecosistemas de bosque seco, como a manglares (Balaguera-Reina et al., 2010). El estado de conocimiento de P. lotor, por su parte, es todavía pobre (Marín-C et al., 2012).

Los roedores representan el orden más diverso dentro de los mamíferos a nivel global (Burgin et al., 2018), pero los registros en el departamento son escasos y esporádicos. Así, por ejemplo, se señaló recientemente en el Atlántico la presencia de Pattonomys semivillosus, una especie endémica de Colombia, de la que se sabe muy poco y que no se registraba para el departamento desde 1949 (Patton et al., 2015; Mejía-Correa, 2018). Probablemente por el bajo esfuerzo de muestreo y la falta 
de exploración en diversas áreas del departamento, la diversidad de roedores y de mamíferos en general podría estar subestimada.

Los primates Saguinus oedipus y Cebus versicolor, especies endémicas del bs- $\mathrm{T}$ y la región Caribe colombiana, se encuentran En Peligro Crítico (CR) y En Peligro (EN), respectivamente (Tabla 2), producto de acciones como la deforestación, la desecación de humedales y el tráfico ilegal (Andrade-C, 2011; de la Torre et al., 2015; Rodríguez et al. 2020). El conocimiento del estado actual de sus poblaciones es incompleto (Martínez, et al. 2010; Rodríguez, et al. 2012), aunque son claves en procesos de dispersión de semillas y polinización (Feilen, et al. 2018; García-Castillo \& Defler, 2018).

La presencia de algunas especies en el departamento es incierta, como es el caso de Sturnira parvidens, que se incluyó en la lista siguiendo a Velazco y Patterson (2014). Igualmente, los roedores Proechimys chrysaelous y Sigmodon hirsutus, y el venado Odocoileus virginianus, que tienen amplia distribución y están presentes en territorios contiguos (Baquero-Montoya \& De la Hoz-Siegler, 2011; Solari et al., 2013; Patton et al., 2015).

La riqueza de las regiones Costera y Canal del Dique puede estar sobreestimada, por el alto número de registros para el municipio de Barranquilla depositados en la Zoologische Staatssammlung München (ZSM), que pudieron ser recolectados en áreas aledañas, como discute Ramírez-Chaves (2014), y asignados a la ciudad capital por ser el área históricamente de mayor tránsito y exportación de productos en el departamento. En cambio, aunque se pensaba que los municipios con influencia del río Magdalena podrían albergar alta diversidad de especies, estos presentan grandes vacíos de información. Se hace necesario, entonces, intensificar muestreos, así como la recolección y revisión de especímenes de museo que permitan ampliar la lista de mamíferos del departamento y establecer criterios claros para el posible establecimiento de nuevas áreas naturales protegidas.

\section{Referencias}

Adler, G. H., Arboledo, J. J. \& Travi, B. L. (1997). Diversity and abundance of small mammals in degraded tropical dry forest of northern Colombia. Mammalia, 61, 361-370.

Alberico, M., Cadena, A., Camacho, J. H. \& Saba, Y. M. (2000). Mamíferos (Synapsida: Theria) de Colombia. Biota colombiana, 1(1), 43-75.
Alexander, T.M., García Q.J., Pino R.J., Posada, A.M. \& Sandoval C.R. (2009). Áreas Protegidas: herramientas de conservación y construcción del territorio en el departamento del Atlántico. Barranquilla, Atlántico: Corporación Autónoma Regional del Atlántico-SIDAP Atlántico-SIRAP Caribe.

http:// www.crautonoma.gov.co/documentos / areasprotegidas / cartillas, \% 20revistas / Cartilla \%20 \% C3\% 81reas\%20Protegidas.pdf.

Allen, J.A. (1916). List of mammals collected in Colombia by the American Museum of Natural History expeditions, 1910-1915. Bulletin of the American Museum of Natural History 35, 191-238.

Alvarado, M. (2016). Sur del Atlántico: una nueva oportunidad. Barranquilla, Atlántico: Fundación Promigas. $372 \mathrm{pp}$.

http:/ / www.fundacionpromigas.org.co/es/Biblioteca/Documents/Libros/Sur\%20del\%20Atlantico\%20-\%20Version \%20Digital.pdf

Andrade-C., M. G. (2011). Estado del conocimiento de la biodiversidad en Colombia y sus amenazas. Consideraciones para fortalecer la interacción ciencia-política. Revista de la Academia Colombiana de Ciencias Exactas, Físicas y Naturales, 35(137), 491-507.

Aya-Cuero, C., Trujillo, F., Mosquera-Guerra, F., Chacón-Pacheco, J., Caicedo, D., Franco-León, N. \& Superina, M. (2019). Distribution of armadillos in Colombia, with an analysis of ecoregions and protected areas inhabited. Mammal Research, 64(4), 569-580.

https:/ / doi.org/10.1007/s13364-019-00435-4

Baquero-Montoya, Á. \& De la Hoz-Siegler, A. (2011). La historia de los Mokaná. Un capítulo de la historia en la región Caribe Colombiana. Revista digital de Historia y Arqueología desde el Caribe colombiano, 14, 232-264

Baker, R. J., Solari, S., Cirranello, A. \& Simmons, N. B. (2016). Higher level classification of phyllostomid bats with a summary of DNA synapomorphies. Acta Chiropterologica, 18(1), 1-38.

https:/ / doi.org/10.3161/15081109ACC2016.18.1.001

Balaguera-Reina, S. A. B., González-Maya, J. F. \& Acero, A. (2010). Fauna nocturna asociada a los manglares y otros humedales en la Vía Parque Isla de Salamanca, Departamento del Magdalena, Caribe colombiano. Boletín de Investigaciones Marinas y Costeras, 39(1), 191-199.

Brown, B. E. (2004). Atlas of New World marsupials. Fieldiana Zoology New Series, 102: 1-308.

https:// doi.org/10.5962/bhl.title.3410

Burgin, C. J., Colella, J. P., Kahn, P. L. \& Upham, N. S. (2018). How many species of mammals are there? Journal of Mammalogy, 99(1), 1-14. 
Caicedo-Herrera, D., Trujillo, F., Rodríguez, C. L. \& Rivera, M. A. (2004). Programa Nacional para la Conservación y Manejo de los Manatíes (Trichechus sp.) en Colombia. Bogotá D.C.: Fundación Omacha-Ministerio de Ambiente, Vivienda y Desarrollo Territorial.170 pp.

Carbone, C., Cowlishaw, G., Isaac, N. J. \& Rowcliffe, J. M. (2005). How far do animals go? Determinants of day range in mammals. The American Naturalist, 165(2), 290-297.

https:/ / doi.org/10.1086/426790

Cardona-Maldonado, M. A. \& Mignucci-Giannoni, A. A. (1999). Pygmy and dwarf sperm whales in Puerto Rico and the Virgin Islands, with a review of Kogia in the Caribbean. Caribbean Journal of Science, 35(12), 29-37.

Castaño, J. H., Saba, Y. M., Botero, J. E. \& Vélez, J. H. (2003). Mamíferos del departamento de Caldas-Colombia. Biota Colombiana, 4(2), 247-259.

Castaño, J. H., Torres, D. A., Rojas-Díaz, V., Saavedra-Rodríguez, C. A. \& Pérez-Torres, J. (2017). Mamíferos del departamento de Risaralda, Colombia. Biota Colombiana, 18(2), 239-254.

Castilla-Consuegra, H. (2013). Anuario estadístico del Atlántico 2013. Barranquilla, Colombia: Secretaría de Planeación Departamental, Subsecretaria de Sistemas de Información y Proyectos, Gobernación del Atlántico. 231 pp.

Chacón-Pacheco, J., Racero-Casarrubia, J. \& Rodríguez-Ortiz, E. (2013). Nuevos registros de Cyclopes didactylus Linnaeus, 1758 para Colombia. Edentata, 14(1), 78-84.

Chacón-Pachecho, J., Figel, J., Rojano, C., Racero-Casarrubia, J., Humanez-Lopez, E., Padilla, H. (2017). Actualización de la distribución e identificación de áreas prioritarias para la conservación de una especie olvidada: el hormiguero gigante en Colombia. Edentata 18, 12-25.

Chacón-Pacheco, J., Avendaño, L. J., Agamez-López, C., Mejía-Fontecha, I. Y., Velásquez-Guarín, D., Ossa, P., Rivera, F., Morales-Martínez, D. \& Ramírez-Chaves, H. E. (2021). Distribution of the dwarf dog-faced bat Molossops temminckii (Chiroptera: Molossidae) in Colombia and comments on its morphometry. Mammalia, 85(2), 182-188.

https:/ / doi.org/10.1515/mammalia-2020-0051

Cirranello, A., Simmons, N. B., Solari, S. \& Baker, R. J. (2016). Morphological diagnoses of higher-level phyllostomid taxa (Chiroptera: Phyllostomidae). Acta Chiropterologica, 18, 39-71.

https:/ / doi.org/10.3161/15081109ACC2016.18.1.002
Corporación Autónoma Regional del Atlántico-CRA. 2013. Estudio de flora y fauna de los municipios de Baranoa y Sabanalarga, Departamento del Atlántico. Barranquilla, Colombia. 372 pp.

Cuervo-Díaz, A., Camacho, J. H. \& Cadena G., A. (1986). Lista actualizada de los mamíferos de Colombia anotaciones sobre su distribución. Caldasia, 15, 471-501.

da Silva, L. G., de Oliveira, T. G., Kasper, C. B., Cherem, J. J., Moraes Jr, E. A., Paviolo, A. \& Eizirik, E. (2016). Biogeography of polymorphic phenotypes: Mapping and ecological modelling of coat colour variants in an elusive Neotropical cat, the jaguarundi (Puma yagouaroundi). Journal of Zoology, 299(4), 295-303.

https:/ / doi.org/10.1111/jzo.12358

de la Torre, S., Morales, A. L., Link, A., Palacios, E. \& Stevenson, P. (2015). Cebus versicolor. The IUCN Red List of Threatened Species 2015: e.T39952A81281674.

https:/ / doi.org/10.2305/IUCN.UK.2015.RLTS. T39952A81281674.en

Delsuc, F., Kuch, M., Gibb, G. C., Karpinski, E., Hackenberger, D., Szpak, P., ... \& Billet, G. (2019). Ancient mitogenomes reveal the evolutionary history and biogeography of sloths. Current Biolo$g y, 29(12), 2031-2042$.

https:// doi.org/10.1016/j.cub.2019.05.043.

Díaz-Pulido, A., Benítez, A., Gómez-Ruiz, D. A., Calderón-Acevedo, C. A., Link, A., Pardo, A., Forero, F., De Luna, G., Payan, E \& Solari, S. (2014). Mamíferos del bosque seco, una mirada al Caribe colombiano. En: Pizano, C y H. García (Eds.) El Bosque Seco Tropical en Colombia (128-165). Bogotá D.C.: Instituto de Investigación de Recursos Biológicos Alexander von Humboldt (IAvH).

Dugand, A. (1947). Aves del departamento del Atlántico, Colombia. Caldasia, 4(20), 499-648.

Feilen, K. L., Guillen, R. R., Vega, J. \& Savage, A. (2018). Developing successful conservation education programs as a means to engage local communities in protecting cotton-top tamarins (Saguinus oedipus) in Colombia. Journal for Nature Conservation, 41, 44-50.

https:/ / doi.org/10.1016/j.jnc.2017.10.003

Ferreira, A. S., Peres, C. A., Bogoni, J. A. \& Cassano, C. R. (2018). Use of agroecosystem matrix habitats by mammalian carnivores (Carnivora): a global-scale analysis. Mammal Review, 48(4), 312-327.

https:/ / doi.org/10.1111/mam.12137

Fundación Yubarta. (2014). Mamíferos marinos migratorios de Colombia. En: Amaya-Espinel, J. D. \& L. A. Zapata (Eds.) Guía de las especies migratorias de la 
biodiversidad en Colombia. Insectos, murciélagos, tortugas marinas, mamíferos marinos y dulceacuícolas. Vol. 3 (29-40). Bogotá D.C.: Ministerio de Ambiente y Desarrollo Sostenible/WWF-Colombia.

García-Castillo, F. \& Defler, T. R. (2018). The diet of Saguinus oedipus in a dry tropical forest and the importance of Spondias mombin Gum as a "fallback food.". Primate Conservation, 32, 67-79.

García, H., Corzo, G., Isaacs, P. \& Etter, A. (2014). Distribución y estado actual de los remanentes del bioma de bosque seco tropical en Colombia: insumos para su gestión. El bosque seco tropical en Colombia. En: Pizano, C y H. García (Eds.) El Bosque Seco Tropical en Colombia (228-251). Bogotá D.C.: Instituto de Investigación de Recursos Biológicos Alexander von Humboldt (IAvH).

Gardner, A. L. (Ed.). (2008). Mammals of South America, Volume 1: Marsupials, Xenarthrans, Shrews, and Bats. Chicago, Illinois: University of Chicago Press. 669 pp.

Gobernación del Atlántico. (2012). Plan departamental de gestión de riesgo del Atlántico. Atlántico, Colombia: Gobernación del Atlántico, Unidad Nacional para la Gestión del Riesgo. Programa de las Naciones Unidas para el Desarrollo Colombia. $125 \mathrm{pp}$.

González-Maya, J. F., Cepeda, A. A., Belant, J. L., Zárrate-Charry, D. A., Balaguera-Reina, S. A. \& Rodríguez-Bolaños, A. (2011). Research priorities for the small carnivores of Colombia. Small Carnivore Conservation, 44, 7-13.

Guzmán, D., Ruíz, J. F. \& Cadena, M. (2014). Regionalización de Colombia según la estacionalidad de la precipitación media mensual, a través análisis de componentes principales $(A C P)$. Bogotá D.C.: IDEAM. 54 pp.

Hernández-Camacho, J., Hurtado-Guerra, A., Ortiz-Quijano, R. \& Walschburger, T. (1992). Unidades biogeográficas de Colombia. En: Halffter, G. (Ed.) La Diversidad Biológica de Iberoamérica I. Xalapa. Programa Iberoamericano de Ciencia y Tecnología para el Desarrollo (105-152). México: Instituto de Ecología A.C. Acta Zoológica Mexicana.

Hershkovitz, P. (1948) Mammals of northern Colombia. Preliminary report No. 2: Spiny rats (Echimyidae), with supplemental notes on related forms. Proceedings of the United States National Museum 97, 125140.

Hershkovitz, P. (1949). Mammals of northern Colombia, preliminary report no. 5: bats (Chiroptera). Proceedings of the United States National Museum, 99, 429454.

Holdridge, L. (1982). Ecología basada en zonas de vida. San José, Costa Rica: Tropical Science Center. 216 pp.
Humanez-López, E., Chacón-Pacheco, J. \& Plese, T. (2015). Áreas de extracción de Xenartros en el Caribe colombiano. Edentata, 16, 65-68.

Instituto de Investigaciones Marinas y Costeras INVEMAR. (2007). Ordenamiento ambiental de la zona Costera del Departamento del Atlántico. Bogotá D.C. Instituto de Investigaciones Marinas y Costeras José Benito Vives de Andreís - INVEMAR. 588 pp

ISA (Interconexión Eléctrica S. A.). (2002).. Inventario de flora, fauna y establecimiento del programa de monitoreo en áreas de jurisdicción de Cardique y Cra. (Informe Técnico). ISA, Fundación Jardín Botánico Joaquín Antonio Uribe de Medellín. Cartagena- Bolívar, 110 pp.

IUCN. (2020). The IUCN Red List of Threatened Species. Version 2020-2.

https: / / www.iucnredlist.org. Downloaded on 09 July 2020.

Jenkins Jr, F. A. (1970). Anatomy and function of expanded ribs in certain edentates and primates. Journal of Mammalogy, 51(2), 288-301.

https:/ / doi.org/10.2307/1378479

Loureiro, L. O., Engstrom, M. D. \& Lim, B. K. (2020). Single nucleotide polymorphisms (SNPs) provide unprecedented resolution of species boundaries, phylogenetic relationships, and genetic diversity in the mastiff bats (Molossus). Molecular Phylogenetics and Evolution, 143, 106690.

https:/ / doi.org/10.1016/j.ympev.2019.106690

Marín-C, D., Ramírez-Chaves, H. E. \& Suárez-Castro, A. F. (2012). Revisión cráneo-dentaria de Procyon (Carnivora: Procyonidae) en Colombia y Ecuador, con notas sobre su taxonomía y distribución. Mastozoología neotropical, 19(2), 259-270.

Martínez, N. J., Cañas, L. M., Rangel, J. L., Barraza, J. M., Montes, J. M. \& Blanco, O. R. (2010). Coleópteros coprófagos (Scarabaeidae: Scarabaeinae) en un fragmento de bosque seco tropical en el departamento del Atlántico, Colombia. Boletín del Museo de Entomología de la Universidad del Valle, 11(1), 21-30.

Mejía-Correa, S. (2018). Nuevos registros e historia natural de Pattonomys semivillosus (I. Geoffroy, 1838) (Rodentia: Echimyidae) en relictos de bosque seco tropical de los departamentos de Bolívar y Atlántico, Colombia. Mammalogy Notes, 5(1), 12-14.

Meza-Joya, F. L., Ramos, E., Cediel, F., Martínez-Arias, V., Colmenares, J. \& Cardona, D. (2018). Predicted distributions of two poorly known small carnivores in Colombia: the greater grison and striped hog-nosed skunk. Mastozoología Neotropical, 25(1), 89-105.

Ministerio del Ambiente y Desarrollo Sostenible. (2017). Resolución 1912 de 2017 “Por la cual se establece el 
listado de las especies silvestres amenazadas de la diversidad biológica colombiana continental y marino costera". Bogotá D. C.

Morales-Martínez, D. (2017). Taxonomía y sistemática de los murciélagos del género Micronycteris Gray, 1982 (Chiroptera: Phyllostomidae) en Colombia. (Trabajo de Grado). Bogotá, Colombia: Universidad Nacional de Colombia, Facultad de Ciencias, Área curricular de Biología, Instituto de Ciencias Naturales.

Morrison, J. C., Sechrest, W., Dinerstein, E., Wilcove, D. S. \& Lamoreux, J. F. (2007). Persistence of large mammal faunas as indicators of global human impacts. Journal of Mammalogy, 88(6), 1363-1380.

https:/ / doi.org/10.1644/06-MAMM-A-124R2.1

Muñoz-Saba, Y. \& Hoyos-R, Y. M. (2012). Los mamíferos del Caribe colombiano. En: Rangel-Ch, J. O. (Ed.). Colombia Diversidad Biótica XII. La región Caribe de Colombia (703-721). Bogotá D.C.: Universidad Nacional de Colombia, Instituto de Ciencias Naturales.

Nicéforo-María, H. (1947). Quirópteros de Colombia. Boletín del Instituto La Salle, Bogotá, 34, 34-47.

Olaciregui, C. (2017). Evaluación de la presencia de 'Marmosa robinsoni' e inventario de mamíferos en el Departamento del Atlántico. Fundación Botánica y Zoológica de Barranquilla. Occurrence dataset

https:/ / doi.org/10.15472/54fglv

Otero, L. V. (2017). Variación espacial de los murciélagos en zonas inundables en el sur del departamento del Atlántico, parte alta subregión Canal del Dique - Colombia. (Trabajo de grado). Puerto Colombia. Atlántico: Universidad del Atlántico, Facultad de Ciencias básicas, Programa de Biología. 41 pp.

Patton, J. L., Pardiñas, U. F. \& D'Elía, G. (Eds.). (2015). Mammals of South America, Volume 2: Rodents. Chicago, Illinois: University of Chicago Press. 1336 pp.

https:/ / doi.org/10.7208/ chicago/9780226169606. 001.0001

Pech-Canche, J. M., Estrella, E., López-Castillo, D. L., Hernández-Betancourt, S. F. \& Moreno, C. E. (2011). Complementarity and efficiency of bat capture methods in a lowland tropical dry forest of Yucatán, Mexico. Revista Mexicana de Biodiversidad, 82(3), 896-903.

http:/ / doi.org/10.22201/ib.20078706e.2011.3.683

Plese, T., Reyes-Amaya, N., Castro-Vásquez, L., Giraldo, S. \& Feliciano, O. (2016). Distribution and current state of knowledge of Hoffmann's two-toed sloth (Choloepus hoffmanni) in Colombia, with comments on the variations of its external morphological traits. Therya, 7(3), 407-421.

http:/ / doi.org/10.12933/therya-16-412
Prado, J. R. \& Percequillo, A. R. (2013). Geographic distribution of the genera of the Tribe Oryzomyini (Rodentia: Cricetidae: Sigmodontinae) in South America: patterns of distribution and diversity. Arquivos de Zoologia, 44(1), 1-120.

https:/ / doi.org/10.11606/issn.2176-7793.v44i1p1-120 Quintero-Toro, C. (2012). Birds of empire, birds of nation: A history of science, economy, and conservation in United States-Colombia relations. Bogotá D.C.: Ediciones Uniandes-Universidad de los Andes. 187 pp.

Racero-Casarrubia, J., Ballesteros-Correa, J. \& Pérez-Torres, J. (2015). Mamíferos del departamento de Córdoba-Colombia: historia y estado de conservación. Biota Colombiana, 16(2), 128-148.

Ramírez-Chaves, H. E. (2014). Mammals of Colombia deposited at the Zoologische Staatssammlung Muenchen, Germany. Biota Colombiana, 15(1), 104-114.

Ramírez-Chaves, H. E. \& Noguera-Urbano, E. A. (2010). Lista preliminar de los mamíferos (Mammalia: Theria) del departamento de Nariño, Colombia. Biota Colombiana, $11(1$ y 2$)$

Ramírez-Chaves, H. E. \& Pérez, W. A. (2010). Mamíferos (Mammalia: Theria) del departamento del Cauca, Colombia. Biota Colombiana, 11(1 y 2), 142-171.

Ramírez-Chaves, H. E., Noguera-Urbano, E. A. \& Rodríguez-Posada, M. E. (2013). Mamíferos (Mammalia) del departamento de Putumayo, Colombia. Revista de la Academia Colombiana de Ciencias Exactas, Físicas y Naturales, 37(143), 263-286.

Ramírez-Chaves, H. E., Suárez-Castro, A. F. \& González-Maya, J. F. (2016). Cambios recientes a la lista de los mamíferos de Colombia. Mammalogy Notes, 3(1), 1-9.

Rangel, J.O, Aguirre-C., J. \& Rodríguez C. L. (2012). La biodiversidad de municipios del Caribe de Colombia. Bogotá D.C.: Ministerio de Ambiente y Desarrollo Sostenible-Universidad Nacional de Colombia, Instituto de Ciencias Naturales, 409-688.

Riba Ingenieros. (2003). Inventario de flora y fauna de los municipios de Polonuevo, Sabanagrande y Galapa. Corporación Autónoma Regional del Atlántico. Barranquilla. Colombia. 257 p.

Rincón, N. M. \& Romero, A. J. (2012). Cetáceos presentes en el Caribe nororiental colombiano (2004-2012). Revista Mutis, 2(2), 60-75.

Ripple, W. J., Estes, J. A., Beschta, R. L., Wilmers, C. C., Ritchie, E. G., Hebblewhite, M., Berger, J., Elmnhagen, B., Letnic, M., Nelson M. P., Schmitz, O. J., Smith, D. W., Wallach, A. D. (2014). Status and ecological effects of the world's largest carnivores. Science, 343(6167), 1241484.

http:/ / doi.org/10.1126/science.1241484 
Ritchie, E. G., Elmhagen, B., Glen, A. S., Letnic, M., Ludwig, G. \& McDonald, R. A. (2012). Ecosystem restoration with teeth: what role for predators? Trends in ecology \& evolution, 27(5), 265-271.

https:/ / doi.org/10.1016/j.tree.2012.01.001

Rodríguez, G. M. \& Banda-R., K., editores. (2011). Plan de Manejo Ambiental del Distrito de Manejo Integrado DMI Luriza - Usiacurí, Atlántico (Informe Técnico). Barranquilla, Colombia: Fundación Ecosistemas Secos de Colombia.

Rodríguez, G. M. \& Banda-R., K., editores. (2012). Plan de Manejo Ambiental de la Reserva Forestal Protectora El Palomar - Piojó, Atlántico (Informe Técnico). Barranquilla, Colombia: Fundación Ecosistemas Secos de Colombia.

Rodríguez, G. M., Banda, K., Reyes, S. P. \& González, A. C. E. (2012). Lista comentada de las plantas vasculares de bosques secos prioritarios para la conservación en los departamentos de Atlántico y Bolívar (Caribe colombiano). Biota Colombiana, 13(2), 7-39.

Rodríguez, V., Defler, T.R., Guzmán-Caro, D., Link, A., Mittermeier, R.A., Palacios, E. \& Stevenson, P.R. (2020). Saguinus oedipus. The IUCN Red List of Threatened Species 2020: e.T19823A115573819.

https:/ / doi.org/10.2305/IUCN.UK.2020-2.RLTS. T19823A115573819.en

Rodríguez-Mahecha, J. V., Alberico, M., Trujillo, F. \& Jorgenson, J. (2006). Libro Rojo de los mamíferos de Colombia. Bogotá D.C.: Conservación Internacional Colombia, Ministerio de Ambiente, Vivienda y Desarrollo Territorial. 443 pp.

Rojas-Díaz, V., Reyes-Gutiérrez, M. \& Alberico, M. S. (2012). Mamíferos (Synapsida, Theria) del Valle del Cauca, Colombia. Biota Colombiana, 13(1), 99-116.

Rubio, V. (2017). Evaluación de composición de ensambles de murciélagos para tres tipos de manejo, en el bosque seco tropical del Caribe colombiano. (Trabajo de grado). Armenia, Colombia: Universidad del Quindío, Facultad de Ciencias Básicas y Tecnologías, Programa de Biología. 24 pp.

Ruiz-García, M., Pacheco, L. F. \& Álvarez, D. (2009). Caracterización genética del puma andino boliviano (Puma concolor) en el Parque Nacional Sajama (PNS) y relaciones con otras poblaciones de pumas del noroccidente de Sudamérica. Revista Chilena de Historia Natural, 82(1), 97-117.

http:/ / doi.org/10.4067/S0716-078X2009000100007

Ruiz-García, M., Parra, A., Romero-Aleán, N., Escobar-Armel, P. \& Shostell, J. M. (2006). Genetic characterization and phylogenetic relationships between the Ateles species (Atelidae, Primates) by means of DNA microsatellite markers and craniometric data. Primate Report, 73, 3-47.

Salamanca-León, I. L. (2016). Cetacean distributions in relation to oceanographic parameters in the Pacific and Atlantic oceans, Colombia. (Master's dissertation). Toronto, Canada: University of Toronto, Physical and Environmental Sciences, Environmental Science. 68 pp.

Sarria-Perea, J, A. (2012). Taxonomia e filogenia de algumas espécies de Mazama (Mammalia; Cervidae) da Colômbia. (Tesis Doctoral). São Paulo, Brasil: Universidade Estadual Paulista, Faculdade de Ciências Agrárias e Veterinárias. 67 pp.

Savage, A., Guillén-Monroy, R., Soto-Rojas, L. \& Vega-Abello, J. (2013). El Proyecto Tití: Medidas efectivas para la conservación del tití cabeciblanco (Saguinus oedipus). En: Defler, T. R., Stevenson, P. R., Bueno, M. L. \& Guzmán-Caro, D. C. (Eds.), Primates Colombianos en Peligro de Extinción (23-38). Bogotá D.C.: Asociación Primatológica Colombiana.

Siles, L. \& Baker, R. J. (2020). Revision of the pale-bellied Micronycteris Gray, 1866 (Chiroptera, Phyllostomidae) with descriptions of two new species. Journal of Zoological Systematics and Evolutionary Research, 2020(00), 1-21.

https:// doi.org/10.1111/jzs.12388

Sociedad Colombiana de Mastozoología. (2019). Mamíferos de Colombia. Versión 1.5. Conjunto de datos.

https:/ / doi.org/10.15472/kl1whs

Solari, S., Muñoz-Saba, Y., Rodríguez-Mahecha, J. V., Defler, T. R., Ramírez-Chaves, H. E. \& Trujillo, F. (2013). Riqueza, endemismo y conservación de los mamíferos de Colombia. Mastozoología Neotropical, 20(2), 301-365.

Thrasher, J. D., Barenfus, M., Rich, S. T. \& Shupe, D. V. (1971). The colony management of Marmosa mitis, the pouchless opossum. Laboratory Animal Science, 21(4), 526-536.

Torres-Trujillo, N. T. \& Mantilla-Meluk, H. (2017). Común e ignorado: ausencia de documentación científica del guatín Dasyprocta punctata (Rodentia: Dasyproctidae) en el departamento del Quindío, Colombia. Revista Biodiversidad Neotropical, 7(1), 30-38.

http:/ / doi.org/10.18636/ bioneotropical.v7i1.588

Trujillo, F., Gartner, A., Caicedo, D. \& Diazgranados, M. C. (2013). Diagnóstico del estado de conocimiento y conservación de los mamíferos acuáticos en Colombia. Bogotá D.C.: Ministerio de Ambiente y Desarrollo Sostenible, Fundación Omacha, Conservación Internacional, WWF. 312 pp. 
Universidad del Atlántico-UT. (2006). Componente de mamíferos. Programa departamental de áreas protegidas, SIRAP. Corporación autónoma regional del Atlántico. $80 \mathrm{p}$.

Velazco, P. M. \& Patterson, B. D. (2014). Two new species of yellow-shouldered bats, genus Sturnira Gray,
1842 (Chiroptera, Phyllostomidae) from Costa Rica, Panama and western Ecuador. ZooKeys, (402), 43-46. Yance-Pérez, O. (1990). Análisis semiológico del arte rupestre de "Piedra pintada"; según la organización económica de los Mokaná. Económicas CUC, 18(1), 54-62. 


\section{Anexos}

Anexo 1. Listado de las especies de mamíferos registrados en el departamento del Atlántico, Colombia. Baq, Barranquilla; Bar, Baranoa; Can, Candelaria; Cdc, Campo de la Cruz; Gal, Galapa; Jda, Juan de Acosta; Lur, Luruaco; Mal, Malambo; Man, Manatí; Pco, Puerto Colombia; Pdv, Palmar de Varela; Pio, Piojó; Pol, Polo Nuevo; Pon, Ponedera; Rep, Repelón; Sgr, Sabanagrande; Sla, Sabanalarga; Slu, Santa Lucía; Sol, Soledad; Sto, Santo Tomás; Sua, Suan; Tub, Tubará; Usi, Usiacurí.

\begin{tabular}{llll}
\hline \multicolumn{1}{c}{ Taxón } & \multicolumn{1}{c}{ Municipio } & \multicolumn{1}{c}{ Referencia } & Voucher \\
\hline DIDELPHIMORPHIA & & & \\
\hline Didelphidae & & & \\
\hline $\begin{array}{l}\text { Didelphis marsupialis Linnaeus, } \\
1758\end{array}$ & $\begin{array}{l}\text { Baq, Bar, Gal, Jda, Pol, } \\
\text { Sla, Sgr, Usi }\end{array}$ & $\begin{array}{l}\text { Solari et al. (2013); Olaciregui } \\
\text { (2017); Brown, (2004) }\end{array}$ & $\begin{array}{l}\text { FMNH 70790; } \\
\text { MHNG-MAM-1736.074 }\end{array}$ \\
\hline Marmosa robinsoni Bangs, 1898 & Baq, Jda, Lur, Pco, Usi & $\begin{array}{l}\text { Thrasher et al. (1971); Gardner } \\
\text { (2008); Solari et al. (2013); Olacire- } \\
\text { gui (2017); Díaz-Pulido et al. (2014) }\end{array}$ & $\begin{array}{l}\text { MVZ: Mamm:135240- 135243 } \\
\text { gut (2013) }\end{array}$ \\
\hline
\end{tabular}

\section{CINGULATA}

Chlamyphoridae

$\begin{array}{lll}\text { Cabassous centralis (Miller, 1899) Rep } & \text { UARC-33 }\end{array}$

Dasypodidae

$\begin{array}{lll}\begin{array}{l}\text { Dasypus novemcinctus Lin- } \\ \text { naeus, } 1758\end{array} & \text { Baq, Gal, Jda, Pio, Sgr, } & \begin{array}{l}\text { Solari et al. (2013); Aya-Cuero et al. } \\ \text { Sla, Usi }\end{array}\end{array}$

PILOSA

\section{Bradypodidae}

Bradypus variegatus Schinz, 1825

\section{Choloepodidae}

Choloepus hoffmani Peters, 1858 Baq

Humanez-López et al. (2015);

Plese et al. (2016)
Solari et al. (2013);

Baq, Jda, Lur, Pio, Usi, Humanez-López et al. (2015);

Olaciregui (2017)

\begin{tabular}{lll}
\hline \multicolumn{1}{c}{ Cyclopedidae } & & \\
\hline $\begin{array}{l}\text { Cyclopes dorsalis (Linnaeus, } \\
\text { 1758) }\end{array}$ & Baq & $\begin{array}{l}\text { Solari et al. (2013); Chacón-Pacheco } \\
\text { et al. (2013). }\end{array}$ \\
\hline \multicolumn{1}{c}{ Myrmecophagidae } & & \\
\hline $\begin{array}{l}\text { Myrmecophaga tridactyla Lin- } \\
\text { naeus, 1758 }\end{array}$ & Baq, Pdv, Pon, Sua, & $\begin{array}{l}\text { Jenkins (1970), Chacón-Pacheco et } \\
\text { al. (2017) }\end{array}$ \\
$\begin{array}{l}\text { Tamandua mexicana (Saussure, } \\
\text { 1860) }\end{array}$ & Baq, Pio, Pol, Rep, Usi & $\begin{array}{l}\text { Solari et al. (2013); Ramírez-Cha- } \\
\text { ves (2014) }\end{array}$ \\
\hline
\end{tabular}




\begin{tabular}{|c|c|c|c|}
\hline Taxón & Municipio & Referencia & Voucher \\
\hline \multicolumn{4}{|l|}{ SIRENIA } \\
\hline \multicolumn{4}{|l|}{ Trichechidae } \\
\hline $\begin{array}{l}\text { Trichechus manatus Linnaeus, } \\
1758\end{array}$ & $\begin{array}{l}\text { Baq, Man, Pdv Rep, } \\
\text { Sla, Slu }\end{array}$ & $\begin{array}{l}\text { Solari et al. (2013); Caicedo et al. } \\
\text { (2004); Trujillo et al. (2013) }\end{array}$ & \\
\hline \multicolumn{4}{|l|}{ QUIROPTERA } \\
\hline \multicolumn{4}{|l|}{ Emballonuridae } \\
\hline $\begin{array}{l}\text { Peropteryx macrotis (Wagner, } \\
1843 \text { ) }\end{array}$ & Pio & Solari et al. (2013) & \\
\hline $\begin{array}{l}\text { Rhynchonycteris naso } \\
\text { (Wied-Neuwied, 1820) }\end{array}$ & Lur, Usi & Solari et al. (2013) & \\
\hline $\begin{array}{l}\text { Saccopteryx bilineata (Tem- } \\
\text { minck, 1838) }\end{array}$ & Pio & Solari et al. (2013); Rubio (2017) & $\begin{array}{l}\text { IaVH-Temp-ACC 614, } \\
615,619\end{array}$ \\
\hline $\begin{array}{l}\text { Saccopteryx canescens Thomas, } \\
1901\end{array}$ & Pio & Rubio (2017) & $\begin{array}{l}\text { IAvH-M-9158; IaVH- } \\
\text { Temp-ACC 775, } 607 .\end{array}$ \\
\hline $\begin{array}{l}\text { Saccopteryx leptura (Schreber, } \\
\text { 1774) }\end{array}$ & Lur, Usi & Solari et al. (2013) & \\
\hline \multicolumn{4}{|l|}{ Molossidae } \\
\hline $\begin{array}{l}\text { Eumops glaucinus (J.A Wagner, } \\
\text { 1843) }\end{array}$ & Pio & Solari et al. (2013) & $\begin{array}{l}\text { MHNG-MAM-1917.096 } \\
(1964)\end{array}$ \\
\hline $\begin{array}{l}\text { Molossops temminckii (Burmeis- } \\
\text { ter, 1854) }\end{array}$ & Pon, Rep & Chacón-Pacheco et al. (2021) & UARC-136Q, 137Q \\
\hline $\begin{array}{l}\text { Molossus bondae J.A. Allen, } \\
1904\end{array}$ & Pon & Gardner (2008); Solari et al. (2013) & ROM 68865 \\
\hline $\begin{array}{l}\text { Molossus coibensis J.A. Allen, } \\
1904\end{array}$ & Pon & Gardner (2008); Solari et al. (2013) & ROM 68864 \\
\hline Molossus molossus (Pallas, 1766) & Baq, Slu & $\begin{array}{l}\text { Gardner (2008); Solari et al. (2013); } \\
\text { Otero (2017) }\end{array}$ & $\begin{array}{l}\text { ROM 45529; FMNH } \\
\text { 69527-69548; ICN } 3829\end{array}$ \\
\hline \multicolumn{4}{|l|}{ Mormoopidae } \\
\hline Pteronotus davyi Gray, 1838 & Tub & & IAvH-M-9509 \\
\hline $\begin{array}{l}\text { Pteronotus gymnonotus (Wag- } \\
\text { ner, 1843) }\end{array}$ & Tub & & IAvH-M-9510; 9511 \\
\hline \multicolumn{4}{|l|}{ Natalidae } \\
\hline $\begin{array}{l}\text { Natalus tumidirostris Miller, } \\
1900\end{array}$ & Pio & & IaVH-Temp-ACC 596 \\
\hline \multicolumn{4}{|l|}{ Noctilionidae } \\
\hline $\begin{array}{l}\text { Noctilio albiventris Desmarest, } \\
1818\end{array}$ & Lur, Sla & Solari et al. (2013); Otero (2017) & IAvH-M-9169 \\
\hline $\begin{array}{l}\text { Noctilio leporinus (Linnaeus, } \\
\text { 1758) }\end{array}$ & Slu & Solari et al. (2013); Otero (2017) & \\
\hline
\end{tabular}

Phyllostomidae

Artibeus jamaicensis Leach, 1821 Bar, Jda

ROM-68853; 688567 (Calamar, Bolivar) 


\begin{tabular}{|c|c|c|c|}
\hline Taxón & Municipio & Referencia & Voucher \\
\hline Artibeus lituratus (Olfers, 1818) & Lur, Rep & Solari et al. (2013); Otero (2017) & MCZ-BANGS-8314 \\
\hline Artibeus planirostris (Spix, 1823) & Lur, Rep, Sla, Slu & Otero (2017) & \\
\hline $\begin{array}{l}\text { Carollia brevicauda (Schinz, } \\
\text { 1821) }\end{array}$ & Lur, Rep, Slu & Otero (2017) & \\
\hline Carollia castanea Allen, 1890 & Pio & & $\begin{array}{l}\text { IaVH-Temp-ACC 602, } \\
606,631,638,647\end{array}$ \\
\hline $\begin{array}{l}\text { Carollia perspicillata (Linnaeus, } \\
\text { 1758) }\end{array}$ & Lur, Pco, Pio, Rep, Slu & Solari et al. (2013); Otero (2017) & $\begin{array}{l}\text { IAvH-M-9161; IaVH- } \\
\text { Temp-ACC 604, } 632\end{array}$ \\
\hline \multicolumn{4}{|l|}{$\begin{array}{l}\text { IaVH-Temp-ACC644 ICN } \\
\text { 2087, } 2088\end{array}$} \\
\hline $\begin{array}{l}\text { Desmodus rotundus (É. Geo- } \\
\text { ffroy Saint Hilaire, 1810) }\end{array}$ & Pio, Rep, Sla & Solari et al. (2013) & $\begin{array}{l}\text { IaVH-Temp-ACC 592, } \\
594,608,609,620,621, \\
623,624,627,628,640,646\end{array}$ \\
\hline $\begin{array}{l}\text { Glossophaga longirostris Miller, } \\
1898\end{array}$ & Baq, Pio & Solari et al. (2013) & $\begin{array}{l}\text { IaVH-Temp-ACC591, } \\
\text { 597, 599, 601, 610, 612, } \\
\text { 617, 626, 636, 637, 643; } \\
\text { ROM68851; 68850; } \\
\text { FMNH 69442; ICN } \\
\text { 7809-7814 }\end{array}$ \\
\hline $\begin{array}{l}\text { Glossophaga soricina (Pallas, } \\
\text { 1766) }\end{array}$ & Lur, Pio, Rep, Sla, Slu & Solari et al. (2013) & $\begin{array}{l}\text { IaVH-Temp-ACC } \\
\text { 598, 600, 611, 634, 639, } \\
\text { 641, 642, 645; FMNH- } \\
\text { 153570;153569; 69452; } \\
69451 ; 69450 ; 69449\end{array}$ \\
\hline $\begin{array}{l}\text { Lonchophylla robusta Miller, } \\
1912\end{array}$ & Pio & Rubio (2017) & \\
\hline $\begin{array}{l}\text { Lophostoma silvicolum d'Orbig- } \\
\text { ny, } 1836\end{array}$ & Pio & Solari et al. (2013) & IaVH-Temp-ACC595 \\
\hline $\begin{array}{l}\text { Micronycteris megalotis (Gray, } \\
1842 \text { ) }\end{array}$ & Pio, Pco, Usi & Solari et al. (2013) & $\begin{array}{l}\text { IaVH-Temp-ACC590, } \\
603,622,625,630,633, \\
635\end{array}$ \\
\hline $\begin{array}{l}\text { Gardnerycteris keenani (Hurtado } \\
\& \text { Pacheco }(2014)\end{array}$ & Pio, PCo, Usi & Solari et al. (2013) & $\begin{array}{l}\text { IaVH-Temp-ACC 613, } \\
629\end{array}$ \\
\hline $\begin{array}{l}\text { Phyllostomus discolor (Wagner, } \\
\text { 1843) }\end{array}$ & Pio, Sla, Slu & Solari et al. (2013); Otero (2017) & $\begin{array}{l}\text { IAvH-M-9159, IA- } \\
\text { vH-M-9160; } \\
\text { IaVH-Temp-ACC } 618\end{array}$ \\
\hline $\begin{array}{l}\text { Phyllostomus hastatus (Pallas, } \\
\text { 1767) }\end{array}$ & & Solari et al. (2013) & \\
\hline $\begin{array}{l}\text { Sturnira parvidens } \\
\text { Goldman,1917 }\end{array}$ & Lur, Rep, Sla, Usi & Otero (2017) & $\begin{array}{l}\text { IAvH-M-9508 } \\
\text { IAvH-M-9513 }\end{array}$ \\
\hline Trachops cirrhosus (Spix, 1823) & Pio, Slu & Solari et al. (2013); Otero (2017) & IaVH-Temp-ACC593, 616 \\
\hline $\begin{array}{l}\text { Uroderma convexum (Lyon, } \\
\text { 1902) }\end{array}$ & Lur, Pio, Sla, Slu & Solari et al. (2013); Otero (2017) & MHNG-MAM-1923.040 \\
\hline
\end{tabular}




\begin{tabular}{|c|c|c|c|}
\hline Taxón & Municipio & Referencia & Voucher \\
\hline \multicolumn{4}{|l|}{ Vespertilionidae } \\
\hline Dasypterus ega (Gervais, 1856) & Baq & Solari et al. (2013) & ICN 8003-8006 \\
\hline Rhogessa io Thomas, 1903 & Baq & $\begin{array}{l}\text { Nicéforo-María (1947); Solari et al. } \\
\text { (2013) }\end{array}$ & \\
\hline Myotis nigricans (Schinz, 1821) & Rep & Solari et al. (2013); Otero (2017) & \\
\hline \multicolumn{4}{|l|}{ CARNIVORA } \\
\hline \multicolumn{4}{|l|}{ Canidae } \\
\hline $\begin{array}{l}\text { Cerdocyon thous (Linnaeus, } \\
\text { 1766) }\end{array}$ & Baq, Can, Usi, & $\begin{array}{l}\text { Solari et al. (2013); Olaciregui } \\
\text { (2017) }\end{array}$ & \\
\hline \multicolumn{4}{|l|}{ Mustelidae } \\
\hline Eira barbara (Linnaeus, 1758) & Pio, Pco, Usi & Solari et al. (2013) & \\
\hline Galictis vittata (Schreber, 1776) & Pco, Pio, Pnu & Meza-Joya et al. (2018) & UARC-6ME \\
\hline Lontra longicaudis (Olfers, 1818) & Baq, Sgr & Solari et al. (2013) & \\
\hline \multicolumn{4}{|l|}{ Procyonidae } \\
\hline $\begin{array}{l}\text { Bassaricyon medius (Thomas, } \\
\text { 1909) }\end{array}$ & Baq & & IAvH-M 0749 \\
\hline Nasua narica (Linnaeus, 1766) & Baq & Ramírez-Chaves (2014) & ZSM 1959/146 \\
\hline Potos flavus (Schreber, 1774) & Baq & $\begin{array}{l}\text { Solari et al. (2013); Ramírez-Chaves } \\
\text { (2014) }\end{array}$ & $\begin{array}{l}\text { MHNG-MAM-1120.005; } \\
\text { MSB:Mamm:327337; } \\
\text { MSB:Mamm:327336; } \\
\text { ZSM 1961/36 }\end{array}$ \\
\hline $\begin{array}{l}\text { Procyon cancrivorus (Cuvier, } \\
1798 \text { ) }\end{array}$ & Jda, Pio, Usi & $\begin{array}{l}\text { Solari et al. (2013); Olaciregui } \\
\text { (2017) }\end{array}$ & \\
\hline Procyon lotor (Linnaeus, 1758) & Pco & Solari et al. (2013) & IAvH-M-6017-6018 \\
\hline \multicolumn{4}{|l|}{ Felidae } \\
\hline $\begin{array}{l}\text { Leopardus pardalis (Linnaeus, } \\
\text { 1758) }\end{array}$ & Jda, Pio, Sab, Usi & Solari et al. (2013) & \\
\hline Leopardus wiedii (Schinz, 1821) & Lur, Pco, Tub, Usi & $\begin{array}{l}\text { Solari et al. (2013); Olaciregui } \\
\text { (2017) }\end{array}$ & \\
\hline Panthera onca (Linnaeus, 1758) & Tub & $\begin{array}{l}\text { Solari et al. (2013); Ramírez-Chaves } \\
\text { (2014) }\end{array}$ & ZSM 1959/135 \\
\hline $\begin{array}{l}\text { Herpailurus yagouaroundi (É. } \\
\text { Geoffroy Sant-Hilaire, 1803) }\end{array}$ & Baq, Lur, Pnu, Sla, Tub & $\begin{array}{l}\text { Solari et al. (2013); Ramírez-Chaves } \\
\text { (2014); da Silva et al. (2016) }\end{array}$ & $\begin{array}{l}\text { MHNG-MAM-1112.098; } \\
\text { OMNH-11533; ZSM } \\
\text { 1960/162, 1960/163 }\end{array}$ \\
\hline Puma concolor (Linnaeus, 1771) & Tub & $\begin{array}{l}\text { Solari et al. (2013); Ruiz-García } \\
\text { et al. (2009) }\end{array}$ & \\
\hline \multicolumn{4}{|l|}{ ARTIODACTYLA } \\
\hline \multicolumn{4}{|l|}{ Cervidae } \\
\hline $\begin{array}{l}\text { Mazama americana Erxleben, } \\
1777\end{array}$ & Lur, Pco, Tub & $\begin{array}{l}\text { Cuervo-Díaz et al. (1986); } \\
\text { Sarria-Perea (2012) }\end{array}$ & IAvH-M-7448 \\
\hline
\end{tabular}




\begin{tabular}{|c|c|c|c|}
\hline Taxón & Municipio & Referencia & Voucher \\
\hline \multicolumn{4}{|l|}{ Tayassuidae } \\
\hline Dicotyles tajacu (Linnaeus, 1758) & Lur, Pio & Solari et al. (2013) & \\
\hline \multicolumn{4}{|l|}{ CETACEA } \\
\hline \multicolumn{4}{|l|}{ Balaenopteridae } \\
\hline $\begin{array}{l}\text { Balaenoptera edeni Anderson, } \\
1879\end{array}$ & & $\begin{array}{l}\text { Solari et al. (2013), Fundación } \\
\text { Yubarta (2014) }\end{array}$ & \\
\hline $\begin{array}{l}\text { Balaenoptera musculus (Lin- } \\
\text { naeus, 1758) }\end{array}$ & & $\begin{array}{l}\text { Rodríguez-Mahecha et al (2006), } \\
\text { Fundación Yubarta (2014) }\end{array}$ & \\
\hline $\begin{array}{l}\text { Balaenoptera physalus (Lin- } \\
\text { naeus, 1758) }\end{array}$ & & $\begin{array}{l}\text { Rodríguez-Mahecha et al (2006), } \\
\text { Fundación Yubarta (2014) }\end{array}$ & \\
\hline $\begin{array}{l}\text { Megaptera novaeangliae (Bo- } \\
\text { rowski, 1781) }\end{array}$ & & $\begin{array}{l}\text { Rodríguez-Mahecha et al (2006), } \\
\text { Fundación Yubarta (2014) }\end{array}$ & \\
\hline \multicolumn{4}{|l|}{ Delphinidae } \\
\hline $\begin{array}{l}\text { Globicephala macrorhynchus } \\
\text { Gray, } 1846\end{array}$ & & $\begin{array}{l}\text { Rodríguez-Mahecha et al (2006), } \\
\text { Fundación Yubarta (2014), Sala- } \\
\text { manca-León (2016) }\end{array}$ & \\
\hline Lagenodelphis hosei Fraser, 1956 & & Trujillo et al. (2013) & \\
\hline Pseudorca crassidens Owen, 1846 & & Salamanca-León (2016) & \\
\hline $\begin{array}{l}\text { Sotalia guianensis (Van Béne- } \\
\text { den, 1864) }\end{array}$ & Tub & $\begin{array}{l}\text { Trujillo et al. (2013), Fundación } \\
\text { Yubarta (2014), Salamanca-León } \\
\text { (2016) }\end{array}$ & \\
\hline Stenella attenuata (Gray, 1846) & & $\begin{array}{l}\text { Solari et al. (2013), Fundación } \\
\text { Yubarta (2014); Salamanca-León } \\
\text { (2016) }\end{array}$ & \\
\hline $\begin{array}{l}\text { Stenella frontalis (G. Cuvier, } \\
\text { 1829) }\end{array}$ & & $\begin{array}{l}\text { Solari et al. (2013); Salamanca-León } \\
\text { (2016) }\end{array}$ & \\
\hline $\begin{array}{l}\text { Steno bredanensis (G. Cuvier, } \\
\text { 1828) }\end{array}$ & & Salamanca-León (2016) & \\
\hline $\begin{array}{l}\text { Tursiops truncatus (Montagu, } \\
\text { 1821) }\end{array}$ & Pco & $\begin{array}{l}\text { Solari et al. (2013), Fundación } \\
\text { Yubarta (2014), Salamanca-León } \\
(2016)\end{array}$ & \\
\hline \multicolumn{4}{|l|}{ Physeteridae } \\
\hline $\begin{array}{l}\text { Physeter macrocephalus Lin- } \\
\text { naeus, } 1758\end{array}$ & & $\begin{array}{l}\text { Solari et al. (2013), Fundación } \\
\text { Yubarta (2014), Salamanca-León } \\
(2016)\end{array}$ & \\
\hline $\begin{array}{l}\text { Kogia breviceps (Blainville, } \\
1838 \text { ) }\end{array}$ & & $\begin{array}{l}\text { Cardona-Maldonado \& } \\
\text { Mignucci-Giannoni (1999), } \\
\text { Solari et al. (2013) }\end{array}$ & \\
\hline \multicolumn{4}{|l|}{ PRIMATES } \\
\hline \multicolumn{4}{|l|}{ Aotidae } \\
\hline Aotus griseimembra Elliot, 1912 & Baq, Usi & Ramírez-Chaves (2014) & $\begin{array}{l}\text { ZSM 1958/67; ZSM } \\
\text { 1958/78; ZSM 1958/68; } \\
\text { ZSM 1958/129 }\end{array}$ \\
\hline Atelidae & & & \\
\hline
\end{tabular}




\begin{tabular}{|c|c|c|c|}
\hline Taxón & Municipio & Referencia & Voucher \\
\hline Ateles fusciceps Gray, 1886 & $\mathrm{Baq}$ & Ruiz-García et al. (2006) & \\
\hline $\begin{array}{l}\text { Alouatta seniculus (Linnaeus, } \\
\text { 1766) }\end{array}$ & Lur, Pio, Tub, Usi & Solari et al. (2013) & \\
\hline \multicolumn{4}{|l|}{ Callitrichidae } \\
\hline Saguinus oedipus (Linnaeus, 1758) & Lur, Pio & $\begin{array}{l}\text { Solari et al. (2013); Ramírez-Cha- } \\
\text { ves (2014); Savage et al. (2010) }\end{array}$ & $\begin{array}{l}\text { ZSM 1956/44; ZSM } \\
1959 / 176\end{array}$ \\
\hline \multicolumn{4}{|l|}{ Cebidae } \\
\hline Cebus capucinus (Linnaeus, 1758) & Pio & $\begin{array}{l}\text { Solari et al. (2013); Ramírez- } \\
\text { Chaves (2014) }\end{array}$ & $\begin{array}{l}\text { ZSM 1963/63; ZSM } \\
1963 / 76\end{array}$ \\
\hline Cebus versicolor Pucheran, 1845 & Baq & Ramírez-Chaves (2014) & ZSM 1960/154 \\
\hline \multicolumn{4}{|l|}{ RODENTIA } \\
\hline \multicolumn{4}{|l|}{ Dasyproctidae } \\
\hline Dasyprocta punctata Gray, 1842 & Lur, Rep, Sla, Slu & $\begin{array}{l}\text { Torres-Trujillo \& Mantilla-Meluk } \\
\text { (2017) }\end{array}$ & \\
\hline \multicolumn{4}{|l|}{ Caviidae } \\
\hline $\begin{array}{l}\text { Hydrochoerus isthmius Gold- } \\
\text { man, } 1912\end{array}$ & Baq, Sla & & KUM-89931 \\
\hline \multicolumn{4}{|l|}{ Cricetidae } \\
\hline $\begin{array}{l}\text { Zygodontomys brevicauda (J.A. } \\
\text { Allen \& Chapman, 1893) }\end{array}$ & Sla & $\begin{array}{l}\text { Prado \& Percequillo (2013); Solari } \\
\text { et al. (2013); Patton et al. (2015) }\end{array}$ & \\
\hline \multicolumn{4}{|l|}{ Cuniculidae } \\
\hline Cuniculus paca (Linnaeus, 1766) & Usi & Solari et al. (2013) & \\
\hline \multicolumn{4}{|l|}{ Echimyidae } \\
\hline $\begin{array}{l}\text { Pattonomys semivillosus (I. Geo- } \\
\text { ffroy, 1838) }\end{array}$ & Bar, Lur & $\begin{array}{l}\text { Mejía-Correa (2018); Patton et al. } \\
(2015)\end{array}$ & FMNH 69117 \\
\hline $\begin{array}{l}\text { Proechimys canicollis (J.A. } \\
\text { Allen, 1899) }\end{array}$ & & $\begin{array}{l}\text { Hershkovitz (1948); Solari et al. } \\
\text { (2013); Patton et al. (2015) }\end{array}$ & \\
\hline \multicolumn{4}{|l|}{ Erethizontidae } \\
\hline $\begin{array}{l}\text { Coendou prehensilis (Linnaeus, } \\
\text { 1758) }\end{array}$ & Baq, Lur, Pco, Usi & Ramírez-Chaves (2014) & $\begin{array}{l}\text { ZSM 1957/167; ZSM } \\
\text { 1957/187; ZSM 1956/199; } \\
\text { ZSM 1958/9; ZSM } \\
\text { 1958/20; ZSM 1958/76 }\end{array}$ \\
\hline \multicolumn{4}{|l|}{ Heteromyidae } \\
\hline $\begin{array}{l}\text { Heteromys anomalus (Thomp- } \\
\text { son, 1815) }\end{array}$ & Can, Pio, Usi & $\begin{array}{l}\text { Cuervo-Díaz et al. (1986); Solari } \\
\text { et al. (2013); Patton et al. (2015) }\end{array}$ & \\
\hline \multicolumn{4}{|l|}{ Sciuridae } \\
\hline $\begin{array}{l}\text { Sciurus granatensis (Humbol- } \\
\mathrm{dt}, 1811 \text { ) }\end{array}$ & Baq, Usi, & Solari et al. (2013) & MSB: Mamm:327335 \\
\hline \multicolumn{4}{|l|}{ LAGOMORPHA } \\
\hline \multicolumn{4}{|l|}{ Leporidae } \\
\hline $\begin{array}{l}\text { Sylvilagus floridanus (J.A Allen, } \\
\text { 1890) }\end{array}$ & Can, Rep, Sla & Solari et al. (2013) & $\begin{array}{l}\text { MHNG-MAM-1079.033; } \\
\text { ICN } 2011\end{array}$ \\
\hline
\end{tabular}


Luis José Avendaño-Maldonado

Universidad del Atlántico.

Barranquilla, Colombia.

https:/ / orcid.org/0000-0002-5370-2247

ljavendano@est.uniatlantico.edu.co

Miguel Ángel Camargo-Alarcón

Universidad del Atlántico.

Barranquilla, Colombia.

https: / orcid.org/0000-0003-0970-5077

mangelcamargo@est.uniatlantico.edu.co

\section{Rafael Borja-Acuña}

Universidad del Atlántico.

Barranquilla, Colombia

https:/ / orcid.org/0000-0003-1211-3654

avianuro51@yahoo.es

\section{Julio J. Chacón-Pacheco}

Universidad de Córdoba.

Montería, Colombia

https:/ / orcid.org/0000-0002-7770-3615

jchacon_bio@hotmail.com

Autor para correspondencia

\section{Mamíferos del departamento del Atlántico, Colombia}

Citación del artículo: Avendaño-Maldonado, L. J., Camargo-Alarcón, M. A., Borja-Acuña, R. \& Chacón-Pacheco, J. J. (2021). Mamíferos del departamento del Atlántico, Colombia. Biota Colombiana, 22(2), 108-126.

https:// doi.org/10.21068/c2021.v22n02a06

Recibido: 16 de Julio de 2020

Aceptado: 7 de enero 2021 